\section{Autoantibodies in glaucoma}

\section{By Lauren Martz, Staff Writer}

Researchers at the Unive rsity of Texas Southwestern Medical Center have identified an autoimmune-mediated mechanism that might be responsible for the development of certain cases of normal-pressure glaucoma. ${ }^{1}$ The finding reveals Fas ligand and autoantibodies that target two heat shock proteins as potential drug targets for a subset of the disease that is not well treated by existing drugs.

Glaucoma is characterized by ocular damage due to the loss or degeneration of retinal ganglion cells and their axons. Although high levels of intraocular pressure cause most cases of the disease, as many as $25-30 \%$ of Caucasian patients and $66 \%$ of Japanese patients present with normal pressure, according to Martin Wax, a professor in the Department of Ophthalmology at UT Southwestern. The Glaucoma Research Foundation estimates there are 4 million glaucoma patients in the U.S.

The marketed products and approved surgical strategies for glaucoma only treat high pressure and are thus ineffective at lowering the optic nerve damage and preventing the progressive blindness associated with the normal pressure subset of the disease. ${ }^{2}$

In a paper published in the Journal of Neuroscience, Wax and colleagues said they may have identified the cause for at least some proportion of normal-pressure glaucoma cases: retinal ganglion cell (RGC) death resulting from autoantibodies against heat shock proteins.

Previous work identified high levels of autoantibodies against heat shock protein 27 (Hsp27) and Hsp60 in patients with normal-pressure glaucoma but did not elucidate the underlying mechanism of what the autoantibodies do. ${ }^{3}$ Building on those results, Wax's team showed that the autoantibodies are linked to RGC death that is mediated by infiltration of T cells and upregulation of Fas ligand (FasL).

The team immunized rats against Hsp27 or Hsp60, and these rats showed lower RGC and axon density than untreated rats, particularly around the optic nerve. In addition, $\mathrm{T}$ cell infiltration into the retina was significantly higher in immunized rats than it was in control rats that only received an adjuvant ( $p=0.017$ and $p<0.001$, respectively). These findings pointed to $\mathrm{T}$ cell influx as a cause of increased RGC death.

Wax's group also tested the effects of Hsp autoantibodies on RGC death in vitro. T cells drawn from rats that were immunized against
Hsp27 and Hsp60 induced apoptosis in 22\% and 25\% of RGCs, respectively. T cells drawn from rats immunized with bovine serum albumin or incomplete Freud's adjuvant did not induce apoptosis.

In vitro, an anti-FasL antibody decreased RGC apoptosis by about $75 \%$ compared with what was seen in cells that were not treated with the antibody. These findings suggest that FasL is involved in autoantibody-mediated RGC death. Immunized mice also had higher levels of the FasL receptor than nonimmunized mice.

Paul Olson, cofounder and CSO of Potentia Pharmaceuticals Inc., told SciBX that the paper stands out because it shows that inducing a short-term immune response to Hsp molecules can recapitulate the features of the disease. That ability, he said, serves as both validation of the biological process causing the disease and as an experimental model.

Potentia's lead compound is POT-4, a synthetic peptide complement 3 inhibitor that is in Phase I testing to treat age-related macular degeneration (AMD).

\section{Patient population}

The discovery that RGC damage may result from autoimmunity could open the door to new types of therapeutics for currently untreatable patients. An open question is how to identify such patients.

According to Wax, "the percentage of patients actually suffering from an autoimmune disease is probably very small. Autoimmunity seems to affect mostly those with normal pressure in the eye, although it is still possibly a component in patients with elevated pressure."

Wax told SciBX that about 5-10\% of normal-pressure patients may have symptoms due to autoimmunity, which is only about $1-2 \%$ of the entire patient population. The causes of disease in the remaining normal-pressure patients are still unknown, he said.

"The treatment strategy for high-pressure glaucoma is pretty clear: if we can lower the pressure, we can often maintain vision," he added. "It is when the eye pressure is in the normal range that the question remains as to what we can do. Pressure lowering is still useful but it may not be enough."

Wax said patients with normal-pressure glaucoma are treated by lowering their pressure either with drugs, laser or surgery to try to alleviate further damage, but he said neither approach may entirely address the pressure-independent causes of retinal cell death that occur in these patients.

Franz Grus, head of experimental ophthalmology at the University Eye Hospital, reinforced this view. He said that lowering intraocular pressure "is the best we can do now, but it is not a causative treatment for glaucoma," he said.

"As a field, we have been hung up on reducing pressure and haven't looked very much into the relevance of autoimmunity in glaucoma," 


\section{TARGETS \& MECHANISMS}

said Barrett Katz, CEO of Danube Pharmaceuticals Inc. "This might not apply to the majority of patients, but it could be useful in a tightly defined subset."

Determining the size of the subset is important, said Alistair Stewart, VP of commercial research at Allon Therapeutics Inc. "In terms of how many people this type of glaucoma affects, it is hard to say. The standard in glaucoma is that intraocular pressure drives the disease, but in the emergency room, you will see symptomatic patients with normal levels of pressure," he said.

Danube is developing DNB-001, a small molecule with dual intraocular pressure-reducing and tissue-protecting activity. A oral formulation has completed Phase II testing. The company is also pursuing a sustained-release formulation to address the patient compliance issues that complicate treatment.

Allon's lead compound, Al-108, is an eight-amino-acid activitydependent neuroprotective protein in preclinical testing for ocular conditions including glaucoma. Earlier this year, the compound missed the primary endpoint in a Phase IIa trial to treat amnestic mild cognitive impairment but did show significance on secondary endpoints.

\section{Therapeutic possibilities}

The next step is translating the findings into therapies.

Wax said there are four likely modes of intervention to treat autoimmune-related glaucoma: "Riddance of the offending antigen, neutralization of the antigen, elimination of the resulting antibody, or interfering with the signal transduction pathways identified."

Of those, Wax thinks that interfering in the newly identified cellular destruction pathway is a promising option. "We might find that in signal transduction pathways, a modulating agent could be made into a drug. For example, preventing FasL from binding to the death domain receptor FasR could interfere with the cell death," he said.

Potentia's Olson thinks small molecule immunosuppressants delivered directly to the eye could help treat glaucoma caused by autoimmunity.

Danube's Katz suggested that researchers "look to prevent T cell activation or potentially downregulate FasL or FasR. But, this is not quickly or easily translatable to a therapeutic benefit because the direct clinical applicability of the science to glaucoma remains to be proven, in concept."

\section{Other applications}

In addition to the therapeutic options opened up by Wax's work, the findings could provide a new animal model for studying autoimmuneinduced glaucoma and perhaps be expanded to other diseases of the CNS.

"The importance of this paper is twofold: it suggests that modulating the immune system could effectively treat a certain subset of the disease and also that it is possible to generate an experimental autoimmune model for glaucoma for further characterization of the disease," Katz told SciBX.

"What I found very interesting is that the significance of the pathways identified here extends to other disease types as well," said Allon's Stewart. "For example, in neuropathic pain, heat shock proteins are released from neuronal cells as well following damage to induce a local inflammatory response."

Stewart suggested that studying autoimmunity caused by antibodies that target heat shock proteins could therefore be useful in finding treatments for other diseases.

"In terms of future work, I am interested in seeing it expanded from ocular to other indications in the central nervous system space," he added.

Wax told SciBX his findings have not been patented.

Martz, L. SciBX 1(43); doi:10.1038/scibx.2008.1038

Published online Dec. 4, 2008

\section{REFERENCES}

1. Wax, M. et al. J. Neurosci.; published online Nov. 12, 2008; doi:10.1523/JNEUROSCI.3200-08.2008

Contact: Martin B. Wax, University of Texas Southwestern Medical School, Dallas, Texas e-mail: mbw817@yahoo.com

2. McKinnon, S. et al. Am. J. Manag. Care 14, S20-S27 (2008)

3. Tezel, et al. Invest. Ophthalmol. Vis. Sci. 39, 2277-2287 (1998)

\section{COMPANIES AND INSTITUTIONS MENTIONED}

Allon Therapeutics Inc. (TSX:NPC), Vancouver, British Columbia, Canada

Danube Pharmaceuticals Inc., New York, N.Y.

Glaucoma Research Foundation, San Francisco, Calif.

Potentia Pharmaceuticals Inc., Louisville, Ky.

University Eye Hospital, Mainz, Germany

University of Texas Southwestern Medical Center, Dallas, Texas 